\title{
Entendiendo las generaciones: una revisión del concepto, clasificación y características distintivas de los baby boomers, $X$ y millennials
}

\author{
Understanding generations: a review of the concept, classification and distinctives traits \\ among baby boomers, generation $\mathrm{X}$ and millennials
}

\begin{abstract}
RESUMEN: La diversidad generacional que actualmente interactúa en el lugar de trabajo representa un reto para las organizaciones, ya que resulta más complejo el desarrollo de acciones encaminadas a atraer y fidelizar al talento humano. El presente artículo describe el concepto del término "generación" analizando los enfoques de diversos autores. Así mismo, se clasifican los diferentes grupos generacionales que han existido en la sociedad, de acuerdo con la fecha en que emergen y son relevados por la siguiente generación. Finalmente, se incluye una perspectiva resumida de las generaciones Baby Boomer, Generación $X$ y Generación $Y$ o Millennials, haciendo énfasis en aspectos como actitud hacia el trabajo, principales motivantes y sus preferencias en educación. Lo anterior constituye un valioso aporte hacia la comprensión de las características de los grupos generacionales que convergen en el lugar de trabajo, brindando a las organizaciones información útil tanto para el desarrollo de estrategias efectivas para la selección, fidelización y motivación de su fuerza laboral como para aprovechar las fortalezas de cada grupo generacional.
\end{abstract}

Palabras clave: generación; generaciones; baby boomers; millennials; generación $Y$; generación $X$.

JEL: M00, M12, 015.

ABSTRACT: The generational diversity currently interacting in the workplace represents a challenge for organizations as the development of actions aimed to attract and retain the best human talent becomes more complex. This article describes the concept of "generation", analyzing different approaches. It also classifies the various generational groups that have existed in society, according to their emerging date and replacement by next generation. Finally, it does a brief analysis of the Baby Boomer generation, Generation $X$ and Generation $Y$ or Millennials, emphasizing aspects such as attitudes towards work, main motivations and preferences in education. This is a valuable contribution to the understanding of the characteristics of generational groups that converge in the workplace, providing organizations with useful information for the development of effective strategies to select, retain and motivate their workforce, while taking advantage of the strengths of each generational group.

Keywords: Generation; Generations Baby Boomers; Generation X; Millennials; Generation Y.

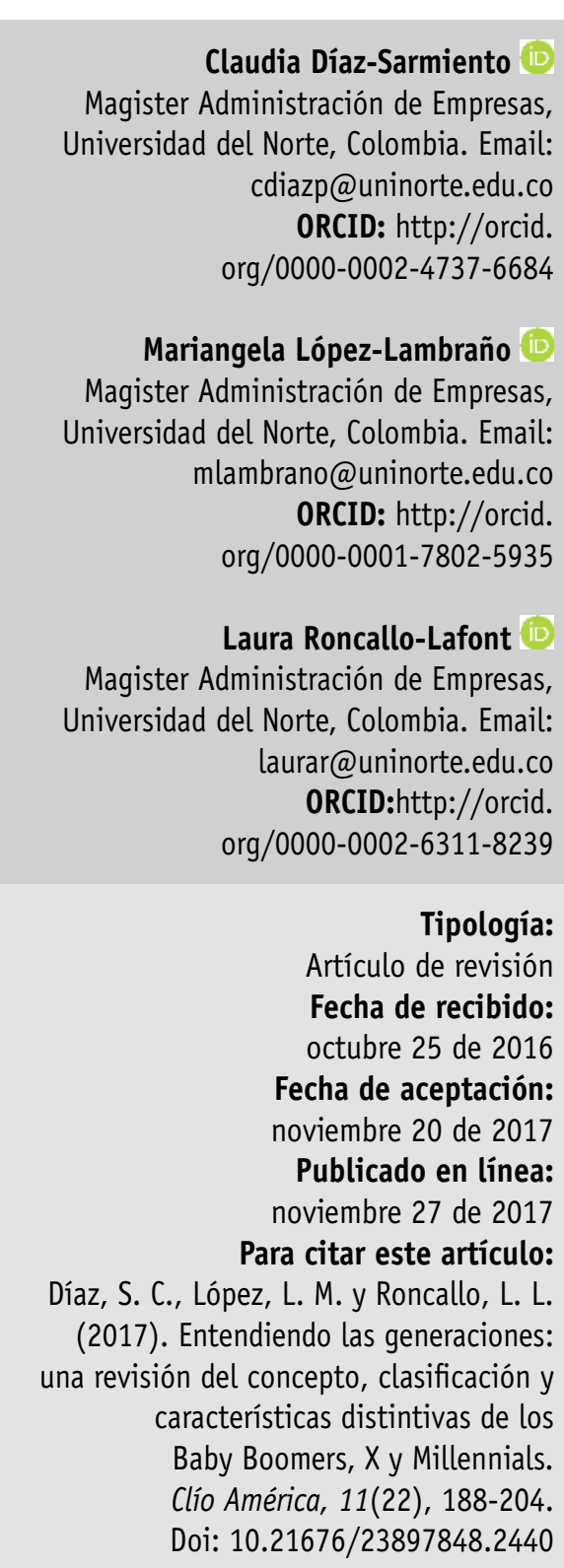




\section{INTRODUCCIÓN}

Administrar la diversidad generacional en el trabajo es uno de los grandes desafíos de la administración moderna en la medida que ésta pluralidad se traduce en diferencias en la concepción del trabajo y en la manera como los individuos entienden y afrontan aspectos como: la autoridad, el liderazgo, el conflicto, la responsabilidad, la comunicación, entre otros. Por lo anterior, el reto de las organizaciones ante la multiplicidad de generaciones en el trabajo es lograr su integración, con el fin de impactar tanto el desarrollo profesional de los individuos como el crecimiento de la compañía.

Aunque el fenómeno de las generaciones ha sido objeto de estudio desde hace décadas por diversos autores y disciplinas, no existe consenso en torno a su definición, elementos determinantes ni a los comportamientos asociados a los grupos generacionales. Lo anterior, complejiza su análisis y dificulta el desarrollo de estrategias que permitan una adecuada gestión de las generaciones en el trabajo.

Dada la importancia del tema, se pretende a través de la presente investigación realizar una revisión descriptiva del concepto de generación, tomando como referencia a la Real Academia Española y a destacados autores que definen el término a la luz de variables que han impactado a los grupos generacionales a lo largo de la historia. El análisis de dicho concepto permite hallar elementos comunes en relación a la duración de una generación, el impacto de la cultura, sociedad y política; para luego clasificar los conceptos hallados en dos grandes vertientes: la visión positivista y la visión histórico romántica.

Lo anterior permitió catalogar a los grupos de generaciones que han sido estudiados frente a las variables trabajo, motivación y educación, como: Tradicionalistas, Generación Silenciosa o Swinger, Baby Boomer Generación X, Generación Millennials o Y, y Centennials.

Finalmente, se describen las tres generaciones que constituyen la fuerza laboral actual: Baby Boomers, Generación X, Generación Millennials o Y, frente a las variables mencionadas anteriormente. Lo anterior aporta a la comprensión de los grupos de humanos que conforman la sociedad actual; siendo esto útil para que las organizaciones desarrollen estrategias que permitan aprovechar las diferencias generacionales en el contexto laboral.

\section{METODOLOGÍA}

Este artículo es un estudio bibliográfico descriptivo fundamentado en una revisión de textos especializados, artículos científicos, de revisión y reflexión y trabajos de investigación sobre el tema generaciones. El estudio se centra en tres elementos fundamentales: la conceptualización del término generación; la clasificación de los grupos generacionales que han hecho parte de nuestra sociedad según el año de aparición y relevo; y la exploración de las generaciones Baby Boomer Generación X, Generación Millennials o Y en relación a las variables trabajo, motivación y educación. La revisión de literatura realizada en el presente artículo se desarrolló con base en las cinco etapas, propuestas por Denyer y Tranfield (2009).

Etapa 1: Formulación de las preguntas de investigación;

Etapa 2: Ubicación de los estudios;

Etapa 3: Selección y evaluación de los estudios;

Etapa 4: Análisis y síntesis; y

Etapa 5: Reporte y uso de los resultados de la investigación.

\section{Etapa 1: Formulación de las preguntas de investigación}

Con la formulación de las preguntas de investigación se da inicio a la revisión bibliográfica, siendo su adecuada estructuración de gran utilidad tanto para la selección y clasificación de los estudios que serán referenciados en la bibliografía como para determinar la información que debe sustraerse de dichos estudios (Counsell, 1997). En ese sentido, las preguntas asociadas a la presente investigación fueron las siguientes:

P1- ¿Qué es una generación?

P2- ¿Cómo han sido clasificados lo grupos generacionales según su año de aparición y relevo? P3- ¿Qué características y/o comportamientos son propios de los individuos pertenecientes a las generaciones Baby Boomer, $X$ y Millennials o $Y$ frente a las variables: trabajo, motivación y educación? 
Entendiendo las generaciones: una revisión del concepto, clasificación y características distintivas de los baby boomers, $x$ y millennials

\section{Etapa 2: Ubicación de los estudios}

Para la localización de la literatura que hace parte de la presente investigación se definieron, en primer lugar, las bases de datos electrónicas que serían consultadas para la ubicación de fuentes relacionadas con el objeto de estudio; estas fueron: Elsevier SD Business Management and Accounting, JSTOR Arts and Sciences VI, EBSCO host Academic Search Complete, Journals@0vid, ProQuest Research Library, Google scholar, Dialnet, Redalyc y Dane. De igual forma, se revisaron textos y publicaciones en medios impresos y electrónicos que permitieron hallar fuentes de gran valor para la investigación.

Seguidamente, se definieron las palabras clave a manera de descriptores de la información a indagar. La palabras clave fueron las siguientes: generaciones, concepto de generación, generaciones en la historia, teoría de las generaciones, clasificación de las generaciones, generaciones y sociedad, evolución histórica de las generaciones, diversidad generacional en el trabajo, generaciones actuales en el trabajo, expectativas y motivaciones de las actuales generaciones, educación y generaciones, convivencia de las generaciones en el trabajo y características de las generaciones Baby Boomer, $\mathrm{X}$ y Millennials o $\mathrm{Y}$.

A continuación, se definió el rango de años para la búsqueda de la información, determinando que para la conceptualización el rango aceptable sería de 1920 hasta 2015; para la clasificación de las generaciones sería entre 2007 y 2016; y para la descripción de las generaciones Baby Boomer, $X$ y Millennials o $Y$ frente a las variables: trabajo, motivación y educación, el rango aceptado sería desde 1989 a 2016. Este rango de fecha se seleccionó a partir de los resultados de la revisión preliminar, la cual arrojó publicaciones de gran relevancia dentro de estos rangos.

Finalmente, se definió que los idiomas admitidos para la búsqueda serían el español e inglés y las áreas de conocimiento serían: ciencias administrativas, ciencias sociales, antropología, psicología, sociología, filosofía e historia.

\section{Etapa 3: Selección y evaluación de los estudios}

Para dar inicio a esta etapa se establecieron como criterios de selección de la bibliografía, la capacidad del estudio para dar respuesta a las preguntas de investigación y la calidad metodológica y científica de la publicación. Se procedió a la revisión de títulos, autores, palabras clave, resumen y conclusiones de los estudios resultantes de la búsqueda preliminar en las bases de datos. Esta revisión permitió realizar una selección de investigaciones entre artículos, tesis, libros, capítulos de libros y otras publicaciones enmarcadas dentro del tema de estudio.

Posteriormente, se efectuó una nueva revisión realizando una lectura crítica de las investigaciones seleccionadas, con el fin de eliminar aquellas cuyo contenido no resultaría útil para los fines de la investigación. Esto permitió disminuir el número de artículos y tener como fuentes bibliográficas aquellas que realmente serían de utilidad para el estudio; seleccionando finalmente 57 publicaciones. En la figura 1 se muestra el número de publicaciones seleccionadas por base de datos.

\section{Etapa 4: Análisis y sintesis}

Para el desarrollo de esta fase se definieron unos criterios para la reunión, síntesis e integración de los estudios seleccionados, haciendo uso de un cuadro de análisis diseñado por las autoras. El cuadro consta de dos secciones: la primera, con información general del estudio, y la segunda con información específica en la que se incluyen datos relacionados con cada una de las preguntas de investigación. (Tabla 1). 


\section{Figura 1.}

Publicaciones seleccionadas por base de datos.

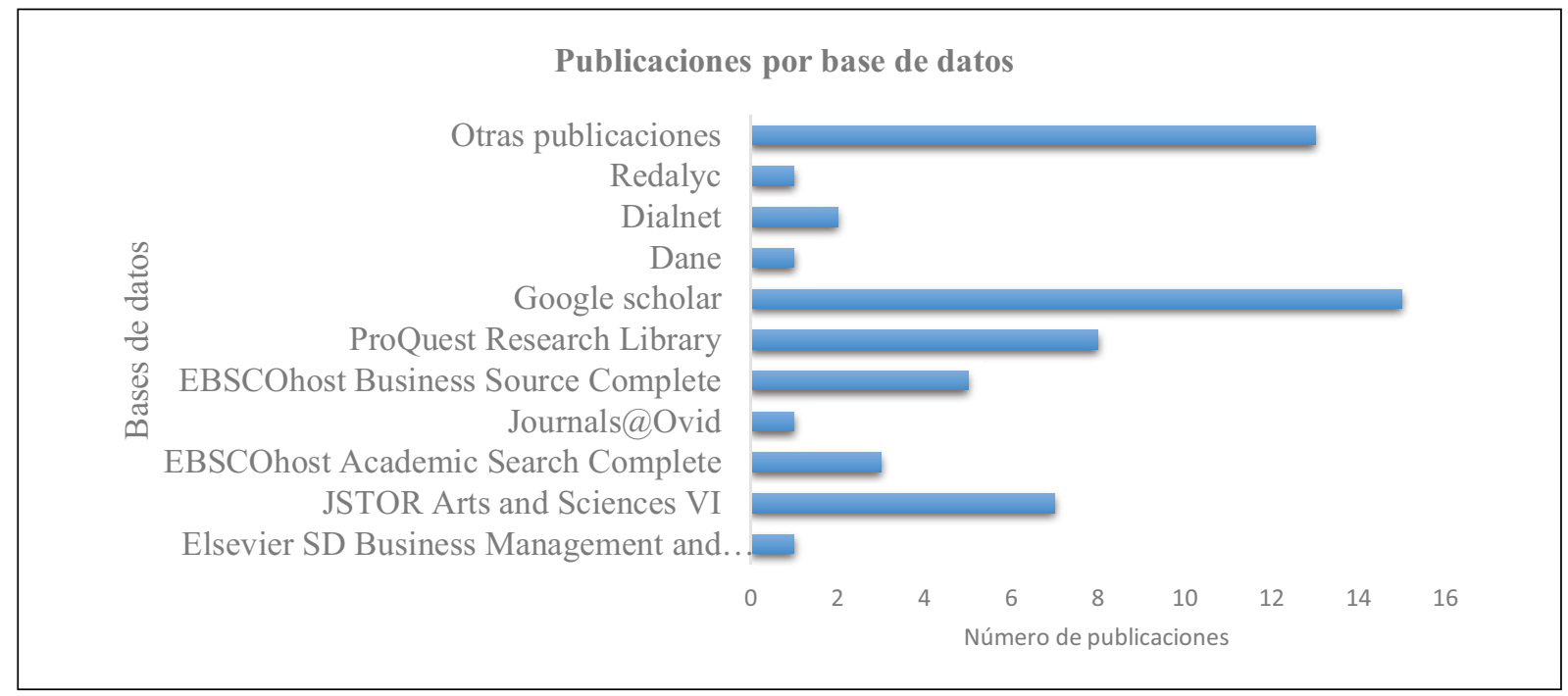

Fuente: elaboración propia.

Tabla 1.

Criterios para la clasificación y análisis de los estudios

\section{INFORMACIÓN GENERAL}

Año

Referencia Bibliográfica

Autor/autores

Nombre del estudio

Descripción del contenido

Área de conocimiento

País

Enfoque de la investigación
Año de publicación de la referencia

Referencia del estudio

Nombre de los autores de estudio

Nombre que el/los autores dieron al estudio

Breve descripción del tema principal del estudio

Disciplina desde la cual se aborda el tema

País en el cual se desarrolla el estudio

Si el enfoque del estudio es cualitativo o cuantitativo 
Entendiendo las generaciones: una revisión del concepto, clasificación y características distintivas de los baby boomers, $x$ y millennials

\section{INFORMACIÓN GENERAL}

Sector al que se aplica

Metodología de investigación del estudio

Base de datos consultada

Tipo de documento

Editorial que publica

Información Específica

Conceptualización

Clasificación

Caracterización Baby Boomers, Generación X y Generación Millennials
Si la investigación se enfoca en algún sector Ej. educación, empresa del sector público o privado,

Si es revisión de literatura, reflexión, encuesta, caso de estudio, estudio científico

Base de datos usada para encontrar el artículo

Si el documento es un journal, memorias, disertación, tesis, libro, entre otros.

Nombre de la casa editorial que publica el artículo.

¿Cómo define el autor el término generación?

¿Cómo clasifica el autor lo grupos generacionales según su año de aparición y relevo?

¿Cómo se caracterizan las generaciones Baby Boomers, Generación X y Generación Millennials; frente a la(s) variable(s): trabajo, motivación y educación?

Fuente: elaboración propia.

\section{Etapa 5: Reporte y uso de los resultados de la investigación.}

Al llegar a esta última etapa ya se ha avanzado en la construcción del estado del arte del tema de investigación, permitiendo a los investigadores tener claridad de lo que se conoce, así como de aquello que aún se desconoce sobre dicha temática. Teniendo la información necesaria para dar respuestas a las preguntas de investigación, es el momento de dar inicio a la redacción del artículo cuidando que exista coherencia en la forma como se estructura el texto. De acuerdo con Bobenrieth (como se citó en Vera, 2009) la redacción de un artículo científico debe tener las siguientes cualidades:
- Claridad: cuando el lector puede leer y comprender el artículo de forma rápida; por lo que es muy importante que la redacción de las ideas esté dispuesta de manera lógica.

- Concisión: cuando las palabras que se usan en la redacción son precisas y pertinentes de tal manera que el lector pueda entender lo que el autor desea transmitir. Para ello deben evitarse el exceso de palabras que pueden llevar al lector a no entender el texto 0 a perderse en el contenido del mismo.

- Precisión: cuando es usado un lenguaje claro con palabras que expresan exactamente lo que se quiere decir. Sencillez y naturalidad: cuando son utilizadas expresiones comunes evitándose el lenguaje complicado y artificial. 


\section{RESULTADOS}

\section{Concepto de generación:}

En la actualidad las organizaciones presentan grandes desafíos en lo relativo a la constitución de su fuerza laboral, debido a la convergencia de múltiples generaciones conviviendo en el lugar de trabajo. El problema se complejiza pues cada generación tiene particularidades y rasgos característicos, diferencias en su fecha de nacimiento, se han enfrentado a contextos culturales, políticos y sociales diferentes y conciben las relaciones con sus empleadores y pares de una forma diferente. Lo cierto es que la brecha generacional actual cada vez es más grande en las empresas, encontrándose diferencias de edades que superan los 20 años en algunos casos, lo cual representa un gran reto para las organizaciones ya que esta diferencia impacta aspectos como comunicación, uso de la tecnología, motivación, reclutamiento, incentivos, entre otros. Por lo anterior, la gestión de las generaciones en el trabajo se ha convertido en un gran desafío para las organizaciones.

Antes de iniciar la descripción de las características y rasgos propios de las generaciones en el trabajo, es necesario definir lo que se entiende por generación. De acuerdo con la definición de la Real Academia Española - RAE (2014) se entiende por generación al "conjunto de personas que, habiendo nacido en fechas próximas y recibida educación e influjos sociales y culturales semejantes, adoptan una actitud en cierto modo común en el ámbito del pensamiento o de la creación". De igual manera 0gg y Bonvalet (como se citó en Chirinos, 2009) definen generación como "un grupo de edad que comparte a lo largo de su historia un conjunto de experiencias formativas que los distinguen de sus predecesores" (p.137). La pregunta obligatoria sería ¿es la edad la que determina la generación a la que se pertenece? 0 ¿son los elementos relacionados con la cultura, hechos históricos e influencias ambientales sus determinantes? 0 ¿Una combinación de todas estas? Lo cierto es que la tarea de entender las generaciones, y acotarlas dentro de un grupo homogéneo, va más allá de ubicarlas dentro de un rango de tiempo en la historia de la humanidad. Autores como Lombardía, Stein y Pin (2008) determinan que para la comprensión del fenómeno generacional es necesario explorar los acontecimientos históricos que llevan a un grupo a tener una convicción compartida sobre la vida y los valores orientadores de su comportamiento.

\section{Visiones asociadas al término generación:}

El concepto de generación ha adquirido diversos matices dependiendo de la óptica de quien lo aborda. Para Kertzer (1983) la palabra generación se asocia con parentesco; con personas que tienen la misma edad y comparten momentos históricos, con etapas de la vida como la juventud, madurez o vejez, entre otras asociaciones. Por su parte, Mannheim y de la Yncera (1993) reducen el espectro de la conceptualización a dos grandes vertientes: una, positivista, que "buscaba captar cuantitativamente los datos básicos del ser-hombre" (p.194) y otra, histórico-romántica, que valora elementos cualitativos. A continuación se describen los planteamientos de algunos autores que, bajo la óptica positivista e histórico-romántica, conceptualizan el término generación.

\section{Concepto de generación bajo la visión positivista:}

Bajo la óptica positivista, Hume (como se citó en Mentré, 1920) establece que el inicio de una generación está ligada a un proceso de cambio de datos que le permite elegir y gobernar de forma diferente a las generaciones anteriores. En ese sentido Mannheim y de la Yncera (1993) resaltan la capacidad y autonomía de las nuevas generaciones para cambiar viejas configuraciones por unas propias, asociadas a su manera particular de ser-hombre.

Por su parte, Comte (como se citó en Leccardi y Feixa, 2011) plantea que las generaciones pueden ser medidas y condensadas dentro del ciclo humano nacimiento-muerte. También establece que las generaciones son instrumentos de medición del progreso en las sociedades; progreso que ocurre y se perpetúa gracias al balance entre los aportes de las nuevas generaciones y la estabilidad brindada por las generaciones pasadas. Para el autor, el tiempo del progreso de las generaciones es de 30 años, al cabo del cual termina y es reemplazado por las nuevas generaciones como en una carrera de relevos. En ese sentido, Mannheim y de la Yncera (1993) plantean que los 
Entendiendo las generaciones: una revisión del concepto, clasificación y características distintivas de los baby boomers, $x$ y millennials

30 años de edad es la etapa en la que el ser humano tiene una mayor capacidad para crear; y los 60, la etapa en la que merma su papel en la vida pública. Es por eso que Leccardi y Feixa (2011) exponen que el progreso puede afectarse cuando el ciclo de vida humana se extiende y las viejas generaciones continúan ocupando un espacio de creación y expresión que ya no les pertenece. Se puede apreciar que los autores citados coinciden en relacionar el término generación con períodos biológicos de la vida humana, así como con cambios en configuraciones sociales que ocurren en el mencionado ciclo vital.

Siguiendo con la revisión bajo la óptica positivista, Ortega y Gasset (1923) plantea que las generaciones están formadas por un "cuerpo social íntegro", relacionado permanentemente para cumplir con un propósito histórico particular. Bajo esa perspectiva Ortega y Gasset (1923) plantea la existencia de ciclos biológicos que condensan por lo menos a tres generaciones; cada una de ellas con características particulares en relación al ejercicio del poder, establecidas de la siguiente manera: Una primera generación constituida por personas que llegan al poder, una segunda formada por las personas que lo ejercen y conservan, y una tercera formada por aquellas que se encuentran en el camino de perderlo. Al respecto, Ortega y Gasset (como se citó en Capell y Niño, 2007), subraya: “Tendríamos entonces «dos etapas distintas de la vida, cada una de quince años: de treinta a cuarenta y cinco, etapa de gestación 0 creación y polémica; de cuarenta y cinco a sesenta, etapa de predominio y mando" (p.71).

Por su parte, Marías (1955) asocia el término generación con una cohorte de seres humanos que, al haber nacido dentro de un rango específico de años, compartirán elementos de tipo social e histórico que los acota dentro de una misma edad. En esa misma línea Mill (como se citó en Marías, 1949) plantea que las generaciones están compuestas por grupos humanos que toman posesión de la sociedad y se encuentran fuertemente influenciados por el momento histórico en el cual se desarrollan. Sumado a lo anterior Mill y Navarro (1969) establecen que dichos grupos humanos, gracias a la educación y experiencia que adquieren, tienen la capacidad de influir en las futuras generaciones para que sean iguales o incluso mejores que ellos.
Asociando el término generación a la vida política, Giusseppe Ferrari (como se citó en Martin, 2015) expone que cada generación tiene una propia concepción del mundo que moldea su pensamiento y actuación en la sociedad. Este autor afirma que cada generación busca implementar su propio pensamiento y convicciones; lo cual, inicialmente, desencadena un período de revolución que luego se disipa en la medida que se estabilice el nuevo orden.

De acuerdo con lo expuesto, se puede apreciar que bajo el enfoque positivista no hay unicidad en el significado que se le atribuye a la palabra generación. Mientras unos autores relacionan el término con grupos humanos que lideran procesos de cambio en la sociedad otros la asocian con individuos que comparten un mismo período biológico, delimitado por el nacimiento y la muerte; algunos autores lo relacionan con una etapa específica del ciclo de las personas como la juventud, madurez o vejez mientras que otros lo asocian con grupos que han sufrido la influencia de acontecimientos propios de un momento de la historia. Lo cierto es que pese a las diferencias en su conceptualización, se observa el interés que comparten los autores por el entendimiento de las características que diferencian los grupos sociales a través de la historia, y el impacto que dichos grupos tienen en la evolución y el progreso de la sociedad.

\section{Concepto de generación bajo la visión histórico romántica:}

La concepción de generación bajo el enfoque Histórico-Romántico se aparta un poco del concepto lineal y de la "sucesión mecánica" tal como lo establece Guisado y Agoiz (2013) para concentrarse en los elementos causantes de cohesionar a los integrantes de una generación.

Dilthey (como se citó en Martin, 2015) define generación como un grupo de personas que viven en un tiempo común, en el cual se comparten conductas y costumbres que los identifica y los hace sentirse cercanos en diferentes facetas de su vida. Su idea de generación y de la conexión que une a los individuos con una generación en particular son los hechos, circunstancias y acontecimientos que se suscitan en su época; siendo estos los que ocasionan que, 
los individuos de una misma generación, puedan responder a situaciones de manera similar. El autor resalta que debido a que ese grupo humano vive el mismo momento de la historia y recibe la misma influencia cultural, política y social tendrá entonces una estructura de comportamiento, valores y moral semejante. Por esto, lo que da la esencia a las generaciones, no es su tiempo cronológico sino su tiempo vivencial que construye un ser interior idénticamente determinado. Como ejemplo de lo anterior, Jaeger (1985) señala que la influencia de los distintos estilos y escuelas de pensamiento de poetas, artistas y filósofos se deriva del momento histórico que viven, más no de su momento biológico. Se puede decir que la gran diferencia con el pensamiento humanista es que no se circunscribe a un espacio de tiempo medible sino que sólo pueden entenderse como algo puramente cualitativo. En este sentido, Dilthey (como se citó en Marías, 1949) añade que: "Generación es además una denominación para una relación de contemporaneidad de individuos; aquellos que en cierto modo crecieron juntos, es decir, tuvieron una infancia común, una juventud común, cuyo tiempo de fuerza viril coincidió parcialmente, los designamos como la misma generación" (p. 60).

Por su parte, Heidegger (como se citó en Del Moral, 2001) fortaleció el concepto generación indicando que se caracterizan por compartir un "destino colectivo". Agrega que los individuos consideran verdaderos aquellos "tópicos históricos comunes" compartidos por la sociedad, los cuales actúan como barreras cuando se desea salir de lo que denomina "el surco trazado por el tiempo". Con lo anterior, el autor explica el poder de la costumbre y la barrera que representa para las nuevas generaciones cuando éstas desean modificar las configuraciones ya establecidas.

\section{Generaciones en la sociedad:}

Actualmente son cinco las generaciones que componen nuestra sociedad (The Center for Generational Kinetics, 2016). Estas generaciones son:

- Tradicionalistas, Generación silenciosa o Swingers.

- Baby Boomers.

- Generación X.

- Generación Y o Millennials

- IGen, Generación Z o Centennials
Dependiendo de las características específicas del mercado laboral, en cada país conviven entre cuatro o cinco generaciones simultáneamente.

Si bien esta categorización es transversal en todo el mundo, la fecha exacta y características de cada generación pueden variar según la ubicación geográfica. Zemke, Raines y Filipczak (2013) definen a las generaciones según su año de nacimiento de la siguiente forma: Generación Y o Millennials: nacidos entre 1980 y 2004, Generación X: nacidos entre 1960 y 1980, Baby Boomers: nacidos entre 1943 y 1960, tradicionalistas o Generación silenciosa: nacidos antes de 1943, inclusive. Según New Strategist Publication (2010) y The Center for Generational Kinetics (2016) los nacidos a mediados de los 90, desde 1996, hacen parte de la generación Z.

A continuación se analizarán las tres generaciones con mayor presencia en el mercado laboral: los Baby Boomer, X y Y o Millennials, sus principales características, eventos de impacto y visión hacia el trabajo.

\section{Generación Baby Boomers}

Actualmente están en sus 50, 60 y hasta 70 años de edad y son definidos por autores como (Roberts y Manolis, 2000; 0sbannon, 2001; Smola y Sutton, 2002) como aquellos nacidos entre 1946 y 1964. Todavía con presencia activa en las organizaciones y principalmente en cargos de poder, los miembros de esta generación son aquellos nacidos recién terminada la segunda guerra mundial; en una época caracterizada por el disparado incremento en el nacimiento de niños: el Baby Boom, especialmente en países anglosajones. Las personas nacidas en este rango de tiempo, actualmente comprenden cerca del $16,8 \%$ de la población Colombiana (Departamento Administrativo Nacional de Estadística - DANE), 2016). De acuerdo con los datos del United States Census Bureau (2016), la población nacida entre 1946 y 1964, compone cerca del $18 \%$ de la población mundial. Esta generación se caracteriza por su dedicación y hasta adicción al trabajo. Empoderados y esperando lo mejor de la vida, es una generación preocupada por la búsqueda de estatus, la lealtad y la calidad de vida.

Debido a su tamaño, desde su nacimiento, los Boomers fueron por mucho tiempo el centro de 
Entendiendo las generaciones: una revisión del concepto, clasificación y características distintivas de los baby boomers, $x$ y millennials

atención del mercado. Estrategias de mercadeo y comercialización estuvieron basadas en esta generación y sus motivaciones de consumo. Este gran grupo generacional tuvo una enorme influencia en la economía y cultura americana durante su época y aún constituyen una fuerte influencia en la actualidad (New Strategist Publication, 2010).

De acuerdo con New Strategist Publication (2010), los Boomers fueron criados por madres jóvenes, en su mayoría amas de casa, tradicionalistas y conservadoras. Fueron enseñados a ser independientes y a creer que podían controlar su destino. Entre sus fortalezas laborales se encuentran la capacidad de guiar, generar y ser flexibles a los cambios (Kupperschmidt, 2000). Están muy preocupados por la participación y el espíritu en el trabajo, en poner corazón y humanizar la vida en la oficina, así como en crear ambientes igualitarios para todos. De acuerdo con Zemke et al., (2013), "ellos son, después de todo, la generación de los derechos civiles, del empoderamiento y la búsqueda de la diversidad".

Algunos eventos que impactaron a esta generación fueron la guerra de Korea, la expansión de la televisión en los hogares, el rock and roll, la guerra de Vietnam, el primer hombre en la luna, el movimiento de los derechos civiles, los derechos de la mujer, el movimiento hippie, woodstock, el asesinato de Kennedy (Zemke et al., 2013). Fue una generación inclinada a la protesta en contra del poder y el activismo a favor de causas sociales.

Esta generación se encuentra proyectándose hacia el proceso de retiro del mercado laboral, algunos ya pensionados, muchos interesados en seguir trabajando después del retiro, otros en seguir formándose 0 alcanzado un mayor estatus a través de títulos de postgrado, y aquellos experimentados que aplican su conocimiento como consultores en diferentes compañías. (Juergensmeyer y Anheier, 2012).

En la actualidad, aquellos activos laboralmente ocupan lugares de poder y están muy bien establecidos profesionalmente en las organizaciones en las cuales construyeron su carrera. En estas son vistos como una pieza esencial para el necesario proceso de transferencia de liderazgo y autoridad a las generaciones siguientes: los $\mathrm{X}$ y Millennials (Juergensmeyer y Anheier, 2012).
A pesar de que crecieron sin internet y todas las herramientas tecnológicas de la actualidad, esta generación ha sido flexible y diligente para aprovechar los beneficios de herramientas como el teléfono móvil y las redes sociales para facilitar su trabajo y su vida personal.

Hawley (como se citó en Juergensmeyer y Anheier, 2012) resalta el beneficio que los Boomers representan para las organizaciones: capacidad para usar su experiencia y visión de largo plazo en la solución de problemas, eliminar el negativismo en el trabajo y negociar mejor que sus colegas más jóvenes. Si bien es cierto que las generaciones más jóvenes se destacan por su capacidad para trabajar en equipo y por su experto manejo de redes sociales, los Boomers son ejemplo de responsabilidad y confiabilidad. (Juergensmeyer y Anheier, 2012)

\section{Generación X}

Muchas son las discrepancias en cuanto al año exacto en que inicia y termina la generación $X$. Según autores como Robert y Manolis (2000); Kupperschmidt (2000) esta inicia partir de los 60 y termina a finales de los 70 e inicios de los 80 .

Definido por la mayoría de autores como aquellos nacidos entre 1965 y 1976 y/o 1977 (Roberts y Manolis, 2000; Smola y Sutton, 2002; New Strategist Publication, 2010), con un límite superior según 0)bannon (2001), hasta el año 1981.

Tomando como base el rango de 1965 a 1981, esta generación se encuentra actualmente en las edades de 35 hasta 51 años de edad. Preceden a los Baby Boomers y son padres de los Millennials y de los Centennials. Actualmente en cargos de mando medio y alto, los $X$ crecieron bajo la sombra de los Baby Boomers (Zemke et al., 2013) y fueron protagonistas del consumismo de los 80 .

De acuerdo con el rango de referencia, los $X$ comprenden actualmente cerca del $18 \%$ de la población Colombiana (Dane, 2016). Según el United States Census Bureau (2016), la población entre 35 y 51 años componen cerca del $21 \%$ de la población mundial. Crecieron en un hogar en donde ambos padres trabajaban o estaban divorciados (Smola y Sutton, 
2002) por lo que fueron formándose en un entorno de inseguridad familiar, altamente cambiante y diverso. Crecieron con ideas liberales, sin una afiliación política particular y con la idea de que la educación superior era el camino a un empleo digno y valioso (Coupland, 1989). Caracterizados por ser una generación cínica y desconfiada en sus mejores épocas; una generación materialista y consumista que no pudo igualar el éxito económico de sus padres (Kupperschmidt, 1998).

En su mayoría fueron los adolescentes durante la época de los 80, influenciados por eventos como el surgimiento de los computadores personales, la expansión del VIH, la expansión del internet, la muerte de John Lennon, Chernobyl, la caída del muro de Berlín, el surgimiento de canales icónicos como MTV y CNN, entre otros eventos que marcaron sus características y comportamiento (Zemke et al., 2013).

Individualistas, pero con altas inclinaciones hacia la búsqueda del apoyo grupal y mentoría (Smola y Sutton, 2002). La necesidad de independencia y de crecimiento profesional de sus padres formó una generación práctica, con una visión pragmática de la realidad (Kupperschmidt, 1998).

Los $X$ ingresan al mercado laboral en los 80 , encontrándose con un mercado en declive, inestable y competido. La seguridad laboral y la creencia de que el crecimiento estaba en la fidelidad hacia una misma compañía pierden valor para esta generación que ve el trabajo como un medio para conseguir sus objetivos, más no como el fin en sí.

A diferencia de los Boomers, los $X$ ven el trabajo solo como trabajo y buscan un balance entre su vida personal y laboral (Marshall, 2004). Mientras que los Boomers buscaban alcanzar su máximo potencial, los $X$ están interesados en sus tres comidas y un techo en donde dormir (Zemke et al., 2013). Los $X$ trajeron consigo características de valor para el mercado laboral. Su alta competencia tecnológica, aceptación a la diversidad y al cambio, se presentan como ventajas para solucionar problemas de la organización de manera efectiva.

De acuerdo con el estudio de Smola y Sutton (2002), lo valores hacia el trabajo de los $X$ difiere significativamente de los Boomers. Ellos se preguntan cuál es su beneficio en cada trabajo y se esfuerzan tanto por alcanzar sus metas, así como las de la organización. En su etapa más joven, Smola y Sutton (2002) encontraron que eran menos leales a la organización y más orientados en su beneficio personal. Sin embargo, el $X$ de la actualidad es un profesional maduro, bien preparado, con responsabilidades y que compone gran parte del mercado laboral. Presenta características como las mencionadas por Gross y Scott (2001), una generación para la cual el trabajo hace parte esencial en su autodefinición y que si bien resiste el paradigma de lealtad corporativa, valora el reconocimiento y retroalimentación de sus jefes así como las relaciones con sus colegas dentro de la organización. Están cansados de las visiones y misiones escritas por sus antecesores (Zemke et al., 2013), y se enfocan más bien en plasmar sus motivaciones y proyecciones en el trabajo.

Actualmente tienen hijos adolescentes, Millennials, con los cuales son protectores y por lo cuales lo dejarían todo. Buscan darles el sentido de familia y estabilidad que no tuvieron, entregándoles el mundo en sus manos y "un mejor futuro".

\section{Generación Y o Millennials}

La generación $\mathrm{Y}$ o Millennials son aquellos nacidos a principios de los 80 e inicios del siglo XXI. Tomando como base el rango propuesto por Zemke et al., (2013), los Millennials son aquellos nacidos entre 1980 y 2004 y están entre sus 10 y 30 años de edad. Sin embargo, al igual que con el resto de generaciones, las fechas exactas de inicio y fin de esta generación varían entre los diferentes autores; principalmente se debate sobre el límite del fin de la generación Y y el inicio de la generación Z o Centennials. Es así como las consultoras PWC (2011), Deloitte (2014), y autores como Cuesta, Ibáñez, Tagliabue y Zangaro (2009), Sprague (2008) y Tulgan (como se citó en Burkus, 2010), se refieren a los Millennials como aquellos nacidos entre 1980 y el año 2000. De acuerdo con De Hauw y De Vos (2010) y Burke y Ng. (2006), el rango va entre 1980 y 1994. Mientras que para Benckendorff, Moscardo y Pendergast (2010), los Millennials son aquellos nacidos entre 1977 y 2003.

Los Y son los hijos de los últimos Boomers y de los primeros $X, y$ crecieron en una cultura de niños protegidos y queridos (Zemke et al., 2013); carac- 
Entendiendo las generaciones: una revisión del concepto, clasificación y características distintivas de los baby boomers, $x$ y millennials

terizados por el uso de la tecnología como parte integral de su estilo de vida. Su afinidad por el mundo digital es uno de los aspectos más destacables de este grupo (PWC, 2011). Han crecido con el internet, los teléfonos inteligentes, acelerados avances tecnológicos, las redes sociales y, con estas, la información al instante. Para ellos la tecnología no es sorprendente, es una obviedad. De acuerdo con esta firma consultora esta es la primera generación que entra al mercado laboral con mayor y mejor conocimiento de las herramientas de negocios que sus superiores. Actualmente en Colombia la población entre 10 y 30 años de edad componen cerca del $40 \%$ de la población (DANE, 2016), mientras que a nivel mundial componen más del $40 \%$ de la población (United States Census Bureau, 2016).

Los Millennials fueron marcados por eventos como el ataque del 11 de septiembre al World Trade Center, la popularidad de google, youtube, wikipedia, redes sociales como facebook y twitter, entre otras, la guerra de Irak y de Afghanistan, el Tsunami de Asia, la recesión del 2008, Ms. office como parte de la vida académica y laboral.

Este grupo generacional ciertamente disfrutó, y disfrutan, de las bondades de las conquistas de la anterior generación, pero con ellos surgieron también nuevas ideas, otras maneras de hacer las cosas. La Generación $Y$ tiene un pensamiento y actuación diferente, es por esto que ha suscitado tanto interés entre las empresas e investigadores de la actualidad. Estos quieren cambiar el mundo, ser más correctos, más honestos, más ecológicos, más orgánicos, más exitosos, pareciera que entran a competir por ser mejores que sus padres. De acuerdo con Stein (2013), los Millennials han tenido tantos galardones y trofeos durante su crecimiento, que muchos de ellos piensan que deben ser promovidos en sus trabajos cada dos años sin importar su rendimiento.

Son ciudadanos de un mundo globalizado; para ellos bien podría ser la casa de al lado, quieren ir y venir para tener una experiencia multicultural, en muchos casos financiada por sus padres. Gracias a la globalización, las características de esta generación son más similares entre países que las de cualquier otra generación (Stein, 2013). Así mismo, son abiertos a la diversidad en todos sus niveles.

El desapego a cualquier afiliación política, así como a las instituciones como la iglesia, es otra de las características de esta generación. De acuerdo con el Pew Research Center (2014), son la generación con menos afiliación política o religiosa de la historia. Están conectados por las redes sociales y tienen una aptitud natural por las vías de comunicación electrónica (PWC, 2011).

Los Millennials son parte importante del mercado laboral en el mundo, muchos están incursionando o establecidos en cargos de liderazgo y algunos otros todavía como practicantes. De acuerdo con el estudio de Deloitte (2014), los Millennials expresan muy poca lealtad con sus empleadores y están constantemente pensando en irse de la compañía en búsqueda de nuevas oportunidades. Según PWC (2011), esta generación prioriza sus intereses personales a los de la compañía. No están cómodos con estructuras rígidas y demandan posiciones variadas e interesantes en el trabajo. Necesitan constante retroalimentación y posibilidad de crecimiento personal y profesional.

En el aspecto familiar, los Millennials conforman hogares en los cuales la pareja es activa laboralmente, por lo cual la flexibilidad en el trabajo es una de sus prioridades. De acuerdo con el estudio de la firma EYGM Limited (2015), los Millennials combinan su ambición y deseo de crecimiento con la necesidad de un trabajo flexible.

A continuación se muestra una perspectiva resumida de las generaciones, enfatizando en aspectos como el trabajo, motivación, educación y rasgos generacionales en las Tablas 2, 3 y 4. 
Tabla 2.

Cuadro comparativo: Baby Boomer, X y Millennials frente a la variable motivación.

\begin{tabular}{|c|c|c|c|}
\hline & BABY BOOMER & $x$ & MILLENNIALS \\
\hline \multirow{3}{*}{ Motivación } & $\begin{array}{l}\text { Les motiva el poder y altos nive- } \\
\text { les de desarrollo. (Cervetti, 2014). } \\
\text { Soñaban con contratos indefini- } \\
\text { dos. (Lasheras y Jiménez, 2012). }\end{array}$ & $\begin{array}{l}\text { Valoran altamente la búsque- } \\
\text { da del balance entre trabajo } \\
\text { y la vida (Bridgers y Johnson, } \\
2006) .\end{array}$ & $\begin{array}{l}\text { Impacientes e innovadores, deman- } \\
\text { dan balance entre trabajo y su auto } \\
\text { interés, dispuestos a sacrificar ga- } \\
\text { nancias financieras a cambio de cosas } \\
\text { significativas. (Seaton y Boyd, 2007) }\end{array}$ \\
\hline & $\begin{array}{l}\text { Perspectiva optimista. } \\
\text { Ante la autoridad expresan amor } \\
\text { u odio. } \\
\text { Espíritu de automotivación. } \\
\text { (Lombardía, Stein y Pin , 2008) }\end{array}$ & $\begin{array}{l}\text { Perspectiva escéptica. } \\
\text { Presentan desinterés ante la } \\
\text { autoridad. } \\
\text { Son equilibrados. } \\
\text { Liderazgo por competencia y } \\
\text { espíritu de anticompromiso. } \\
\text { (Lombardía, Stein y Pin, 2008) }\end{array}$ & $\begin{array}{l}\text { Lo repetitivo, fácil o monótono les } \\
\text { preocupa más que el cómo hacer- } \\
\text { lo; Les gusta el reto. (Lasheras y } \\
\text { Jiménez, 2012). }\end{array}$ \\
\hline & $\begin{array}{l}\text { Autosuficientes, independientes y } \\
\text { interesados en riquezas materia- } \\
\text { les. (Seaton y Boyd, 2007) }\end{array}$ & & $\begin{array}{l}\text { Mayor desempeño en entornos } \\
\text { creativos, les gusta viajar y desen- } \\
\text { volverse en su ambiente de trabajo } \\
\text { (Chirinos, 2009). Son ciudadanos del } \\
\text { mundo. (Cervetti, 2014) }\end{array}$ \\
\hline
\end{tabular}

Fuente: elaboración propia

Tabla 3.

Cuadro comparativo: Baby Boomer, $\mathrm{X}$ y Millennials frente a la variable educación.

\begin{tabular}{|l|l|}
\hline \multicolumn{1}{|c|}{ BABY BOOMER } \\
\hline $\begin{array}{l}\text { Educación como medio de } \\
\text { progreso: "soy lo que soy en } \\
\text { el trabajo". (Cervetti, 2014). }\end{array}$ \\
\hline Educación & $\begin{array}{l}\text { Educación necesaria para el } \\
\text { éxito (Lasheras y Jiménez, } \\
\text { 2012). }\end{array}$ \\
\hline & $\begin{array}{l}\text { Forman parte de lo que es } \\
\text { hoy la vida política, cultural, } \\
\text { industrial y académica en los } \\
\text { Estados Unidos. (Arias, 2011) }\end{array}$ \\
\hline
\end{tabular}

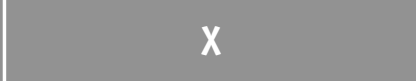

Estudian, se capacitan, nada es garantía para progresar, aprenden idiomas (Cervetti, 2014).

Profesionales de alto nivel, interesados por mantener su rango socioeconómico (Chirinos, 2009)

Nivel de compromiso con el aprendizaje y educación durante toda la vida. (University of Michigan, 2013)

\section{MILLENNIALS}

Contemplan oficios y carreras profesionales no tradicionales, con sistemas como la educación virtual. (Ferreiro, 2006)

Excelente formación académica. (Lasheras y Jiménez, 2012).

Piden cambios y cuestionan la Escuela tradicional, debido al cambio del mercado laboral. (Batalla, 2016) 
Entendiendo las generaciones: una revisión del concepto, clasificación y características distintivas de los baby boomers, $x$ y millennials

\section{Tabla 4.}

Cuadro comparativo: Baby Boomer, X y Millennials frente la variable trabajo.

\begin{tabular}{|c|c|c|c|}
\hline & BABY BOOMER & $x$ & MILLENNIALS \\
\hline \multirow{4}{*}{ Trabajo } & $\begin{array}{l}\text { Acostumbrados a trabajar en } \\
\text { entornos jerárquicos y com- } \\
\text { petitivos. El trabajo a presión } \\
\text { es su estilo de gestión. } \\
\text { (Lasheras y Jiménez, 2012). }\end{array}$ & $\begin{array}{l}\text { Buscan equilibrio entre } \\
\text { lo laboral y lo personal } \\
\text { (Chirinos, 2009). }\end{array}$ & $\begin{array}{l}\text { Buscan flexibilidad laboral (Chirinos, } \\
\text { 2009). Placer y diversión en el trabajo } \\
\text { (Molinari, 2011). Poca lealtad con los } \\
\text { empleadores, nuevas oportunidades, } \\
\text { y posiciones variadas en el trabajo. } \\
\text { (Deloitte, 2014) }\end{array}$ \\
\hline & $\begin{array}{l}\text { Dispuestos a sacrificar la fa- } \\
\text { milia por el trabajo (Bridgers } \\
\text { y Johnson, 2006). }\end{array}$ & $\begin{array}{l}\text { El trabajo es algo temporal } \\
\text { y cada empresa un escalón } \\
\text { para alcanzar algo mejor. } \\
\text { (Filipczak, 1994) }\end{array}$ & $\begin{array}{l}\text { Buscan un balance entre el trabajo y } \\
\text { la familia. (Yeaton, 2008). }\end{array}$ \\
\hline & $\begin{array}{l}\text { Dedicación al trabajo, bús- } \\
\text { queda de estatus, mejora en } \\
\text { el nivel de vida y orientación } \\
\text { al trabajo como ancla de vida } \\
\text { (Almeida, 2012). }\end{array}$ & $\begin{array}{l}\text { Se preguntan cuál es su be- } \\
\text { neficio en cada trabajo y se } \\
\text { esfuerzan tanto por alcan- } \\
\text { zar sus metas, así como las } \\
\text { de la organización. (Smola } \\
\text { y Sutton, 2002). }\end{array}$ & $\begin{array}{l}\text { Expectativas frente al trabajo se cen- } \\
\text { tran en: } \\
\text { La libertad para tomar decisiones. } \\
\text { Las oportunidades de aprendizaje y } \\
\text { desarrollo. } \\
\text { La comunicación abierta y el respeto } \\
\text { por su estilo de vida. (Lombardía, Stein } \\
\text { y Pin, 2008) }\end{array}$ \\
\hline & $\begin{array}{l}\text { Disciplinados, con confianza } \\
\text { en el sistema, manejan la for- } \\
\text { malidad y autoridad (Lasheras } \\
\text { y Jiménez, 2012). }\end{array}$ & & $\begin{array}{l}\text { Independientes, delegan responsabi- } \\
\text { lidades, demandan retroalimentación } \\
\text { inmediata y esperan un sentido de } \\
\text { resultados cada hora Martin (como } \\
\text { se citó en Irizarry-Hernández y de } \\
\text { Arecibo, 2009) }\end{array}$ \\
\hline
\end{tabular}

Fuente: elaboración propia

\section{CONCLUSIÓN}

Al explorar el tema de las generaciones, desde su conceptualización hasta el entendimiento de las características de las generaciones que actualmente conforman la fuerza laboral, resultan evidentes las marcadas diferencias de estos grupos humanos en cuanto a conductas, expectativas, motivaciones, entre otros. En el plano laboral, la administración de la diversidad generacional es un desafío para las organizaciones tanto por la dificultad de la gestión del talento humano con edades distintas como para el diseño de mecanismos para la atracción, promoción y fidelización de trabajadores pertenecientes a diferentes generaciones.

Teniendo en cuenta la importancia del estudio de las generaciones, se inicia explorando el concepto de generación. De esta forma, se puede concluir que esta palabra es concebida de manera distinta, dependiendo del enfoque y perspectiva de los autores que estudiaron este fenómeno social. El análisis de 
las variables incluidas en las definiciones permitió situar los aportes conceptuales en dos líneas de pensamiento: una positivista y otra histórico-romántica. La positivista relaciona la palabra generación con grupos humanos con elementos comunes en relación a su ciclo biológico, siendo variables como la edad o la etapa (juventud, madurez o vejez) las que condicionan su pertenencia a una determinada generación. Estos grupos humanos viven un momento histórico común y tienen la capacidad de revolucionar la sociedad, imponiendo su propio pensamiento. La visión histórico-romántica afirma que la pertenencia a una generación depende de la influencia histórica, política, social y cultural que reciben los individuos.

Por su parte, la indagación bibliográfica permitió obtener registros de cinco generaciones en la historia que han sido objeto de estudio por diversos autores; los Tradicionalistas (conocidos también como Generación silenciosa o Swingers), los Baby Boomers, la Generación X, la Generación Y o Millennials, y la IGen, Generación Z o Centennials. Se observó que la fecha exacta de inicio y terminación de cada generación varía con el enfoque utilizado por cada autor y la influencia que para este tienen elementos como el entorno económico, político, social, cultural, tecnológico, entre otros.

Adicionalmente, este estudio permitió analizar las principales características de las generaciones que se encuentran activas en el mercado laboral. Se encontró que esta caracterización se fue construyendo a partir de las marcadas diferencias, manifestadas con respecto a la generación anterior, la cuales se hacen evidentes cuando la nueva generación entra en la etapa de adolescencia y al mercado laboral. Es decir, cada generación fue forjando su percepción de la siguiente generación a partir del conflicto generado por las diferencias en el entorno familiar, académico y profesional. El estudio de los Baby Boomers es el más completo, hasta ahora, en relación con el análisis de la generación en sus diferentes etapas desde la adolescencia hasta su madurez y actual retiro del mercado laboral. Así mismo se evidencia, a través de los diferentes estudios, la consolidación de la generación $X$ en su vida personal y profesional, demostrando cambios como una mayor lealtad y compromiso con en el trabajo a medida que avanza hacia la etapa adulta. Se demuestra, a su vez, el espacio para un análisis a futuro, sobre su proceso de transición hacia su retiro laboral. Finalmente, se analiza el nacimiento de la generación Millennial y su avance desde la adolescencia hasta el ingreso al mercado laboral, basando entonces la caracterización actual en una etapa joven de dicha generación. Se espera que los futuros estudios profundicen en la etapa de madurez personal, académica y profesional de la generación Millennial, así como en su relacionamiento e influencia sobre la generación Centennial.

De igual forma, fue posible evidenciar como las vivencias, experiencias y características de cada generación, fueron influenciando e incluso moldeando los principales valores del siguiente grupo generacional. La gran dedicación al trabajo y al crecimiento profesional por parte de los Baby Boomers se tradujo en una menor dedicación a su entorno familiar, generando a su vez, una generación $X$ inclinada hacia la búsqueda de un equilibrio entre lo familiar y lo personal; todavía interesados en el crecimiento profesional inculcado por sus predecesores, pero viendo el trabajo con un medio para conseguir sus objetivos y no como objetivo final. Este profundo interés por no descuidar el entorno familiar, como hicieron sus padres se reflejó en una actitud protectora de su familia y sus hijos, pertenecientes a la generación Millennial, quienes crecieron en un entorno de completa atención, con el mundo a sus pies; evidenciando posteriormente una marcada necesidad de retroalimentación, sentimiento de derecho y necesidad de promoción. Así mismo el desarrollo tecnológico, la globalización y los acelerados cambios en el mercado laboral, impulsados en gran medida por la intensa actividad productiva de las pasadas generaciones, constituyó el entorno en el que se fueron formando los Millennials, dando espacio para el desarrollo de la innovación, creatividad y apertura mental que caracteriza a esta generación.

La investigación realizada sirve como punto de partida para estructurar futuras líneas de investigación asociadas con el tema, que permitan seguir aportando al conocimiento de las generaciones en relación a variables distintas a las analizadas en el presente estudio. La mayoría de los estudios realizados a nivel mundial describen características de la generación $X$ 
Entendiendo las generaciones: una revisión del concepto, clasificación y características distintivas de los baby boomers, $x$ y millennials

y $Y$ principalmente con respecto a variables como: proyección personal, valores, percepción de la autoridad, liderazgo y compromiso organizacional. Sin embargo hay un vacío en estudios que permitan caracterizar a las generaciones $X$ y $Y$ en Colombia, particularmente en la Región Caribe colombiana, con respecto a variables como la educación y el trabajo, para determinar si los rasgos propios de esta población son equiparables a los rasgos generacionales de otras sociedades.

Conviene que las empresas y la sociedad se preparen para hacer el relevo generacional, de tal forma que aprovechen la ventaja competitiva que brinda tener varias generaciones conviviendo en el mismo lugar para no perder el conocimiento que brinda la experiencia de los Baby Boomers y aprovechar los conocimientos, ímpetu, preparación y juventud de los Millennials. Brindar a nivel empresarial la oportunidad de enseñar y aprender unos de otros a nivel generacional puede ayudar a romper las barreras que se crean en las relaciones laborales por la convivencia generacional.

\section{REFERENCIAS BIBLIOGRÁFICAS}

Almeida, G. M. E. (2012). Trayectoria profesional de la generación de los" Baby Boomers". Universidad Andina Simón Bolivar Sede Ecuador, Quito, Ecuador.

Arias, S. (2011). La generación de los "baby boomers". ¿Quiénes son los "baby boomers"?. EEEUU, California: Psicológicamente Hablando. Recuperado de http://www.psicologicamentehablando.com/ la-generacion-de-los-baby-boomers/

Batalla, J. (16 de Julio de 2016). Millennials y educación: cómo sus usos culturales crean un nuevo paradigma [Mensaje en un blog]. Recuperado de http://www.infobae.com/tendencias/2016/07/16/ Millennials-y-educacion-como-sus-usos-culturalescrean-un-nuevo-paradigma-educativo/

Benckendorff, P., Moscardo, G. y Pendergast, D. (2010). Tourism and generation $Y$. Wallingford: CABI Publishing.

Burke, R. J. y Ng, E. (2006). The changing nature of work and organizations: Implications for human resource management. Human Resource Management Review, 16(2), 86-94.
Burkus, D. (2010). Developing the next generation of leaders: How to engage Millennials in the workplace. Leadership Advance Online Issue, XIX, 1-6. Recuperado de https://www.regent.edu/acad/global/ publications/lao/issue_19/Burkus_leading_next_generation.pdf

Bridgers, M. y Johnson, H. (2006). The aging workforce: The facts, the fiction, the future. ASHRAE Journal, 48(1), A6-A9.

Capell, H. C. y Niño, E. L. (2007). El método histórico de las generaciones: el caso de la psicología española. Revista de Historia de la Psicología, 28(1), 67-86.

Cervetti, M. D. P. (2014). Conflictos por la convivencia de baby boomers, generación $X$ y generación $Y$ en los equipos de trabajo: equipos de auditoría de Deloitte (Tesis de pregrado). Universidad de San Andrés, Buenos Aires, Argentina.

Coupland, D. (1989). Generation X. Vista. Recuperado de http://joeclark.org/dossiers/GenerationX.pdf

Counsell, C. (1997). Formulating Questions and Locating Primary Studies for Inclusion in Systematic Reviews. Annals of Internal Medicine, 127(5), 380-387.

Cuesta, E. M., Ibáñez, M. E., Tagliabue, R. y Zangaro, M. (2009). La nueva generación y el trabajo. Barbarói, (31), 126.

Chirinos, N. (2009). Características generacionales y los valores. Su impacto en lo laboral. Observatorio Laboral Revista Venezolana, 2(4), 133-153.

Del Moral, J. M. (2001). Historicidad y temporalidad en El ser y el tiempo de M. Heidegger. Signos filosóficos, (5), 133-141.

De Hauw, S. y De Vos, A. (2010). Millennials' career perspective and psychological contract expectations: does the recession lead to lowered expectations?. Journal of Business and Psychology, 25(2), 293-302.

Deloitte, LL. P. (21 de enero de 2014). Big demands and high expectations: What generation $Y$ wants from business, government, and the future workplace [Mensaje en un Blog]. Recuperado de https://www2. deloitte.com/uk/en/pages/press-releases/ archive-press-release/big-demands-and-highexpectations-what-generation-y-wants.html

Departamento Administrativo Nacional de Estadística (DANE). (2016). Estimaciones y proyecciones de la población de Colombia. Recuperado de: https://geoportal.dane.gov.co/v2/?page=elementoEstimaciones.

Denyer, D. y Tranfield, D. (2009). The sage handbook of organizational research methods. Reference and Research 
Book News, 24(3). Recuperado de: https://search. proquest.com/docview/199760922?accountid=41515

EYGM Limited. (2015). Global generations A global study on work-life challenges across generations. Recuperado de http://www.ey.com/Publication/ vwLUAssets/EY-global-generations-a-global-studyon-work-life-challenges-across-generations/\$FILE/ EY-global-generations-a-global-study-on-work-lifechallenges-across-generations.pdf

Ferreiro, R. F. (2006). El reto de la educación del siglo XXI: la generación N. Apertura, 6(5).

Filipczak, B. (1994). It's Just a Job: Generation X at Work. Training, 31(4), 21-27.

Gross, D. y Scott, S. (2001, 24 de Junio). Proceeding with caution. Revista Time. Recuperado de: http://content. time.com/time/magazine/article/0,9171,155010,00. html

Guisado, M. C. y Agoiz, A. B. (2013). ¿Es operativo el concepto de generación? Aposta: Revista de ciencias sociales, (56), 1.

Irizarry-Hernández, E. B. y de Arecibo, R. (2009). La generación y o generación milenaria: El nuevo paradigma laboral. Inter Metro Business Journal, 5(2), 10.

Jaeger, H. (1985). Generations in history: Reflections on a controversial concept. History and Theory, 24(3), 273-292.

Juergensmeyer, M. y Anheier, H. K. (2012). Encyclopedia of Global Studies. Thousand Oaks, California: SAGE Publications, Inc.

Kertzer, D. I. (1983). Generation as a sociological problem. Annual review of sociology, 9(1), 125-149.

Kupperschmidt, B. R. (1998). Understanding generation X employees. Journal of Nursing Administration, 28(12), 36-43.

Kupperschmidt, B. R. (2000). Tips to help you recruit, manage, and keep Generation X employees. Nursing management, 31(3), 58-60.

Lasheras, M. y Jiménez, E. (2012). Generación Y Talento. IESE Business School. Universidad de Navarra. Recuperado de https://www.iese.edu/Aplicaciones/ upload/Generacion.pdf

Leccardi, C. y Feixa, C. (2011). El concepto de generación en las teorías sobre la juventud. Última década, 19(34), 11-32.

Lombardía, P. G., Stein, G. y Pin, J. R. (2008). Políticas para dirigir a los nuevos profesionales-motivaciones y valores de la generación Y. Documento de investigación. DI-753. Recuperado de https://www.iese.edu/ research/pdfs/DI-0753.pdf
Mannheim, K. y de la Yncera, I. S. (1993). El problema de las generaciones. Reis, (62), 193-242.

Marías, J. (1949). El método histórico de las generaciones. Recuperado de http://www.cervantesvirtual.com/nd/ ark:/59851/bmcb8739

Marías, J. (1955). La estructura social teoría y método. Recuperado de http://www.cervantesvirtual.com/nd/ ark:/59851/bmc184z5

Marshall, J. (2004). Managing different generations at work. Financial Executive, 20(5), 18-19.

Martin, M. (2015). La teoría de las generaciones de Ortega y Gasset: una lectura del siglo XXI. Tiempo y espacio, (20), 98-110.

Mentré, F. (1920). Les Générations sociales. Paris: Éditions Bossard, 470, (2) p.

Mill, J. S. y Navarro, M. Á. L. (1969). Ensayo sobre la libertad. Recuperado de http://www.encuentrodefilantropos.co/Textos/Ensayo $\% 20$ sobre $\% 201$ la $\% 20$ Libertad.pdf

Molinari, P. (2011). Turbulencia generacional. Buenos Aires: Temas Grupo Editorial.

New Strategist Publication, I. (2010). American Generations: Who They Are and How They Live. Ithaca, N.Y.: New Strategist Publications, Inc

O'Bannon, G. (2001). Managing our future: The generation X factor. Public Personnel Management, 30(1), 95-110.

Ortega., y Gasset, J. (1923). El tema de nuestro tiempo. Obras completas, 3, 143-203.

Pew Research Center. (2014). Millennials in Adulthood. Recuperado de http://www.pewsocialtrends. org/2014/03/07/Millennials-in-adulthood/

PWC. (2011). Millennials at work Reshaping the workplace. Recuperado de https://www.pwc.com/m1/en/services/ consulting/documents/Millennials-at-work.pdf

Real Academia Española. (2014). Diccionario de la lengua española (23.a ed.). Recuperado de http://dle.rae. es/?id=J3hJP2w

Roberts, J. A. y Manolis, C. (2000). Baby boomers and busters: an exploratory investigation of attitudes toward marketing, advertising and consumerism. Journal of Consumer Marketing, 17(6), 481-497.

Seaton, L. J. y Boyd, M. (2007). The organizational leadership of the post baby boom generation: an upper echelon theory approach. Academy of Entrepreneurship Journal, 13(2), 69-77.

Sprague, C. (2008, febrero). The Silent Generation meets Generation Y: How to manage a four-generation 
Entendiendo las generaciones: una revisión del concepto, clasificación y características distintivas de los baby boomers, $x$ y millennials

workforce with panache. Talent Strategy, Human Capital Institute White Paper. Recuperado de http:// www.theccic.org/Customer-Content/WWW/CMS/files/ Gen_Y_Characteristics.pdf

Stein, Joel. (2013). The Me Me Me generation. Revista Time. Recuperado de http://time.com/247/ Millennials-the-me-me-me-generation/

The Center for Generational Kinetics. (2016). Generational Breakdown: Info About All of the Generations. Austin, Texas: GenHQ. Recuperado de http://genhq.com/ faq-info-about-generations/

United States Census Bureau. (2016). World Population by Age and Sex 2016. Recuperado de https://www. census.gov/population/international/data/worldpop/tool_population.php

Smola, K. y Sutton, C. D. (2002). Generational differences: Revisiting generational work values for the new millennium. Journal of organizational behavior, 23(4), 363-382.

University of Michigan. (2013). La mayoría de las personas de la generación $X$ continúa su educación. Recuperado de https://espanol.umich.edu/noticias/2013/05/07/ la-mayoria-de-las-personas-de-la-generacion-xcontinua-su-educacion/

Vera, C. 0. (2009). Cómo escribir artículos de revisión. Revista Médica La Paz, 15(1), 63-69.

Zemke, R., Raines, C. y Filipczak, B. (2013). Generations at work: Managing the clash of Boomers, Gen Xers, and Gen Yers in the workplace. EEUU: Kindle Edition de AMACOM Div American Mgmt Assn.

Yeaton, K. (2008). Recruiting and managing the'why?'generation: Gen Y. The CPA Journal, 78(4), 68. 